\title{
On the Prediction of Optical Aberrations by Personalized
}

\author{
Eye Models
}

\author{
Rafael Navarro \\ ICMA, Consejo Superior de Investigaciones Científicas-Universidad de Zaragoza, Facultad de \\ Ciencias, Plaza San Francisco s/n, 50009 Zaragoza, Spain \\ e-mail: rafaelnb@unizar.es
}

\section{Luis González}

Instituto Nacional de Técnica Aeroespacial. Ctra. Ajalvir km 4 - Torrejón de Ardoz, 28850

Madrd, Spain

\section{José L. Hernández-Matamoros}

Clínica Oftalmológica Real Visión, Santa Cruz de Marcenado 31, 28015 Madrid, Spain

\begin{abstract}
A method to develop optical models of individual eyes is presented. First we choose a generic eye model as the input guess, and then apply a two-stage customization procedure. Stage 1 consists of replacing, in the initial generic model, those anatomical and optical parameters with experimental data measured on the eye under analysis. In this study, the set of experimental data used was that provided by a standard clinical preoperative exam, namely corneal elevation topography, ultrasound biometry, and total wave aberration. Then, the second stage is to find the unknown lens structure that would reproduce the
\end{abstract}


measured wave aberration through optical optimization. This customization method has been applied to 19 eyes with different degrees of spherical ametropia (from $0.4 \mathrm{D}$ to $-8 \mathrm{D}$ ). For each eye, two totally different initial eye models have been compared: one considers a simpler constant refractive index for the lens, whereas the second model has a GRIN lens. The results were highly satisfactory, with $100 \%$ convergence, and with average RMS prediction errors about $\lambda / 100$. This is one order of magnitude lower than typical measurement errors. Regarding lens geometry, the first model with a constant refractive index lens tended to overestimate surface curvatures, whereas for the second GRIN model with a quadratic index distribution we had the opposite trend, namely surfaces that were too flat. The proposed method is highly efficient and robust giving a high fidelity reproduction of the wavefront in all cases attempted so far. Limitations found in reproducing the geometry of the lens seem to be associated with the use of inaccurate models of its refractive index.

Submitted to OVS, $29 / 1 / 2006$ 


\section{INTRODUCTION}

Theoretical models are necessary to link the anatomy to the optical performance of the eye. They are important to understand the role and relative importance of the optical elements or make predictions on optical and image quality. Nowadays, the design of optimal ablation patterns $^{1,2,3}$ in custom refractive surgery, the selection of intraocular lens implants ${ }^{4}$, or the design of visual optics in general, require to have accurate geometrical and optical models of the human eye.

Traditionally, eye models have been generic in the sense that they represent average anatomical and optical properties. Since the $17^{\text {th }}$ century (Huygens'simplified eye, 1653) generic models have evolved to better represent the optical performance of an average eye (see Ref. 5 for a review). Probably the most successful eye model was proposed by Gullstrand ${ }^{6}$, and later updated by Le Grand ${ }^{7}$. This model is reasonably simple, based on anatomy, and reproduces the Gaussian properties of an average eye. Since then, many alternative eye models have been proposed. On the one hand, reduced eyes ${ }^{8}$ consist of a single refracting surface, but Y. Le Grand pointed out that "it is necessary to avoid the use of the reduced eye because such a scheme is too crude" ${ }^{7}$. These single-surface models do not even consider that the eye has two main optical elements, cornea and lens; thus they are anatomically incorrect and can hardly be used in real applications. Other authors have put the emphasis in following anatomy more accurately, incorporating the most relevant components and structure including the gradient-index (GRIN) distribution of the lens ${ }^{9,10}$. One important problem is that the exact distribution of the refractive index of the human lens ${ }^{11,12,13}$ is not well known yet, and these models tend to have several adjustable parameters. Other schematic models have shown the crucial role of aspheric surfaces in keeping aberrations (both spherical and off-axis) within moderate values, and in being able to 
reproduce the overall optical performance of the average eye ${ }^{14,15}$. This type of schematic eyes, using a constant equivalent refractive index for the lens, have been used as wide angle models ${ }^{16}$, ${ }^{17}$, as well; some of them have included accommodation ${ }^{15}$ or changes with age ${ }^{18}$, and have been applied to different problems such as the design of corneal ablation patterns to correct for spherical aberration $^{19}$. A few attempts have been also made to consider the joint effect of aspheric surfaces and GRIN distribution upon spherical aberration of the lens ${ }^{20}$, or to put all the main features together (aspheric surfaces, GRIN lens, pupil decentration, visual axis) in an anatomical-optical model ${ }^{21}$.

All these generic models have been useful to understand the optics of the human eye, and to design visual optics, but they can strongly differ from real individual eyes. All the above generic models are highly symmetric, having ideal rotationally-symmetric, centered and aligned surfaces, whereas real eyes often show irregularities, with no well-defined optical or symmetry axes. In addition, there is a large intersubject variability, so that individual eyes often show marked anatomical differences from the mean. Therefore, a generic model can only predict the order of magnitude of optical performance parameters, but never give a high fidelity reproduction of the optics of an individual eye. In fact, even normal eyes often show irregular aberrations, both at the fovea ${ }^{22,23}$ and off-axis ${ }^{24}$.

Yet, the challenge today is to develop custom treatments, such as in refractive surgery, and these rely on having a detailed description of both the anatomy and the optical properties of the eye under treatment. In this sense, corneal topographers have enabled the accurate measurement of the corneal surface as well as the development of optical models of the cornea, which have been validated by different authors ${ }^{25,}{ }^{26}$. However, the modeling of the complete optical system of the eye is a much harder problem, mainly because it is difficult to obtain, in 
vivo, precise anatomical data on the internal optical elements. Several studies have focused on the relative contribution of cornea and internal optics to the optical performance ${ }^{27,28}$; and there is a also an increasing number of publications on the in vivo measurement of internal geometry, including radii of curvature and asphericities ${ }^{29}$, surface alignment ${ }^{30}$, as well as changes with age and accommodation ${ }^{31},{ }^{32}$. Nevertheless, the reliability and accuracy of these in vivo measurements are still controversial ${ }^{33,34}$.

In summary, with today's technology we can obtain some partial information about the geometry of internal optics of the eye, but there is still a large uncertainty, mainly about how to model the lens. In general we will not have access either to the 2D topographies of the lens surfaces, or the GRIN structure for the particular eye under study. While promising techniques, such as OCT (optical coherence tomography) ${ }^{35}$ are starting to be applied to the lens, here we study a different strategy. While there is an increasing effort to continue developing better in vivo measurements of the lens geometry and GRIN distribution, here we focus on a complementary way to develop custom eye models. Here we shall assume that the lens geometry is unknown, and the method will consist of finding the lens topography which best reproduces the experimentally measured wave aberration (including both low and high order aberrations), through the optimization of an optical model. Of course, this procedure will not get rid of all uncertainties about the true topography and GRIN of the lens; but at least, we could get a reasonably plausible custom model of the optics of the eye and be able to predict its optical performance, and hence we thought it was worth trying despite these potential drawbacks. A further reason is that, to the best of our knowledge, a tangible implementation and analysis of such a model which is able to predict the optical aberrations of an individual eye has not been reported before. 
Therefore, the primary goal of this work was to develop a method to obtain schematic models of individual eyes which are able to reproduce their optical performance, in particular the monochromatic wave aberration, with high fidelity. Ideally, this kind of model could be used as a simulation, analysis and optimization tool in many applications, from diagnosis and analysis of the outcome of treatments, to the optimization of custom refractive surgery, or the custom design of intraocular lenses, etc. Consequently, we wanted to develop a procedure that was both as simple as possible and fully automatic, under the assumption that the only input to build the eye model (except for control experiments) is the set of anatomical and optical parameters gathered in the scenario of a standard ophthalmology clinic, reasonably well-equipped with diagnostic tools including elevation corneal topography, ultrasound biometry and aberrometry. We depart from a given generic eye model, and the customization is achieved in two main stages. First we replace generic parameters by known data of the eye under study. Then, we perform an optimization in which we find the topographies of the lens which reproduce the total wave aberration (including both refractive state and higher order aberrations, HOA). We want to point out that this numerical procedure does not try to substitute methods based on the direct measurement of the lens optics and geometry, but to complement them.

The specific objectives of this work are the following:

1.- To develop the methodology and study the accuracy achievable in reproducing the total wave aberration in different eyes.

2.- As the number of unknown parameters is large, it is important to study possible alternative solutions compatible with the data. For this purpose, we will compare solutions obtained starting from two different lens models, with constant ${ }^{15}$ and gradient (GRIN) ${ }^{21}$ 
refractive index respectively, or the results obtained when using different optimization procedures.

3.- To study, through a tolerance analysis, what geometrical variables in the lens (radii, decentrations, conic constants, etc.) are more or less critical in the minimization. This is important as it will tell us those parameters that one needs to know more precisely to obtain a reliable model of the eye under study.

4.- To study the robustness and reliability of the method, analyzing drawbacks and identifying the main limitations to guide further research in this crucial question.

\section{METHODS}

Here we address the issue of building a personalized eye model as an optimization problem, similar, to some extent, to those found in classical optical design. Some differences are: Here we have an incomplete set of geometrical data of the eye to be modeled, which will be used as constant parameters during the optimization. Instead of minimizing optical aberrations, here we want to reproduce the experimentally measured aberrations and refractive state (i.e. total wave aberration) of the eye under study. One analogy with optical design is that we will depart from an input guess, or initial model of the optical system, which is then optimized through adaptation to our particular goal. In our case, the input guess is one generic average model eye. To depart from an average is a way to maximize the prior probability of all the parameters in the model, if we have no specific information on the particular the eye to be modeled. Of course, if there are specific data available, these should replace the generic values in the model. Therefore, the main stages of our approach, depicted in Figure 1, are: 
- We depart from a generic schematic eye model (here we consider two different eye models).

- The first customization stage consists of substitution of generic values by the available data of the patient:

i) Anatomical. Elevation topography of the cornea, pupil centre and axial thickness of optical media.

ii) Optical. Total wave aberration, including second and higher order aberrations.

At this stage, it is important to establish the direction of the chief ray, passing through the center of the pupil. Since the corneal topography is referenced to the keratometric axis ${ }^{5}$, and this is almost parallel to the visual axis (in a first rough approximation), we use the approximation that the chief ray is aligned along the $\mathrm{Z}$ axis of the topographer. In other words, the corneal surface is introduced into the model without any rotation or translation. The pupil is placed according to the pupil center provided by the ORBSCAN $\mathrm{II}^{\mathrm{TM}}$ topographer. The rest of the refracting surfaces (back surface of the cornea and lens) are those of the initial generic model and these are also kept also aligned to the keratometric axis. The topography and alignment of the back surface of the cornea are not customized for the sake of simplicity in the procedure. We have computed the impact of different changes in alignment, curvature radii and conic constant of this surface upon the wave aberration, and found it to be almost negligible within most plausible ranges. The thicknesses of the optical media are also replaced with the biometric data.

\#\#\#\#\#\#\#\#\#\# Insert Figure 1 about here \#\#\#\#\#\#\#\#\#\#

Once we have this mixture model of generic and customized elements, then we can perform a preliminary ray tracing to compute the total wave aberration, WA, predicted at this 
initial stage. As we can see in the example of Fig. 1, the predicted HOA (central row, right) are completely different from the measured values (left) in most cases. Some amount of mismatch also occurs in refractive state (or second order aberrations). In fact, the prediction error (right column), given as the difference between the measured and predicted aberrations, can be as large as the WA itself. Thus, it is essential to customize the unknown geometrical parameters, through the optimization stage described below.

\subsection{Generic eye models}

One potentially important aspect is the dependence of the result on the initial generic eye used; as the final custom model will necessary depend on those parameters of the initial model that are not changed during the optimization process. One clear example is the refractive index of the lens. For this reason we have compared two totally different generic models representing constant and GRIN refractive index for the lens, respectively.

The first generic model ${ }^{15}$ has a constant refractive index lens, and is used here in the unaccommodated state. See upper part of Table 1. It was based on the Gullstrand-LeGrand model $^{7}$, with a constant effective refractive index for the lens, but incorporating experimental average asphericities (conic constants) of the refractive surfaces ${ }^{36}$. The radius of the anterior surface of the cornea was updated using published data of the mean shape of the cornea ${ }^{37}$. In addition, the dispersions of the refractive indexes were adjusted to fit the longitudinal chromatic aberration. This model varies continuously with accommodation and reproduced remarkably well the overall average optical performance (aberrations, polychromatic MTF and PSF) of the eye, both on-axis ${ }^{15}$ and off-axis ${ }^{17}$. Its total refractive power is $60.4 \mathrm{D}$. 
The second model ${ }^{21}$ is rather different (see lower part in Table 1). The radii and thickness of the cornea vary only slightly form the above model, but the conic constants are totally different, making the anterior surface somewhat less prolate $(\mathrm{Q}=-0.18)$ but the posterior surface significantly more prolate $(\mathrm{Q}=-0.6)$. This posterior $\mathrm{Q}$ was adjusted to fit the spherical aberration of the model, and considered the possibility that this conic constant yields a more plausible marginal corneal thickness. But the main difference is that the lens is totally different from most previous models. First, they consider the GRIN distribution of the lens, and use a quadratic expression to fit experimental data ${ }^{12}$ :

$$
n(x, y, z)=n_{00}+n_{01} z+n_{02} z^{2}+n_{10}\left(x^{2}+y^{2}\right)
$$

They divide the lens into two anterior and posterior sections by inserting an imaginary plane surface at the equator (see Table 1), and then they apply Eq. 3 to fit the index of each section, anterior and posterior, independently. These anterior and posterior GRIN parameters are given in Table 1, bottom rows. In addition, the lens radii, taken from an in vivo study by Brown ${ }^{38}, 12.4$ $\mathrm{mm}$ and $-8.1 \mathrm{~mm}$ respectively, were considerably larger than in previous classic eye models. Nevertheless, the refractive power of the lens, and hence of the eye $60.35 \mathrm{D}$, was similar to that of the first model, because the GRIN distribution was adjusted to compensate for the decrease in power associated to these flatter surfaces. This model considers that the visual axis is $+5^{\circ}$ offaxis and a pupil decentration of $+0.5 \mathrm{~mm}$. We have adopted the $+5^{\circ}$ angle for the lens in both models, whereas the pupil position has no effect here, since we introduce the experimental value at the first stage of customization.

One especially interesting aspect is the age dependence of the GRIN distribution. Jones et al. ${ }^{39}$ have recently reported an interesting study in which younger lenses have an approximately 
quadratic index distribution, while older lenses (40 years or more) tend to have a basically flat (constant) refractive index in the centre, and much steeper in the periphery. Roughly speaking, model 2 could be more appropriate for younger and model 1 for older subjects respectively.

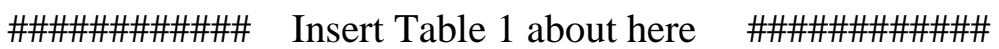

\subsection{Optimization}

The optimization is based on minimizing the wave aberration prediction error. The method was implemented using CodeV ${ }^{\mathrm{TM}}$ (Optical Research Associates, Pasadena CA) optical design software. At this stage, we assume that the refractive indexes are constant among individuals and equal to those of the generic model used. As we discuss later, this is indeed one of the most critical aspects of the procedure, as the result will necessarily depend on the refractive index model used. Nevertheless, further research would be necessary to have accurate refractive index models, since recent studies suggest an important change of the refractive index distribution of the lens with age ${ }^{39}$. Consequently, the remaining unknowns, used as free parameters during optimization, are the topographies of both lens surfaces.

To give a parametric description of the aspheric lens surfaces, we utilize similar expressions to those commonly used to represent corneal topography ${ }^{40}$. Our standard surface will be the sum of two terms, a regular revolution conic surface plus a Zernike polynomial expansion which accounts for departures of the real surface from the regular basis:

$$
Z=\frac{x^{2}+y^{2}}{R+\sqrt{R^{2}-(1+Q)\left(x^{2}+y^{2}\right)}}+\sum_{n} c_{n} Z_{n}
$$


where $z$ is the surface sagitta as a function of the $x, y$ coordinates; $R$ the apical radius of curvature; $Q$ the conic constant; $Z_{n}$ the n-th Zernike polynomial and $c_{n}$ the corresponding coefficient. Here we consider up to $7^{\text {th }}$ order, that is 36 Zernike coefficients (piston included), following the OSA standard notation. Therefore, for each surface we have 38 parameters. The same expression is used for the corneal and lens surfaces. In the case of the anterior corneal surface, we analyze the matrix of sagitta samples provided by the topographer in ASCII format. These data are fitted to Eq. 1 by a least squares method implemented in MATLAB (Mathworks, Mass). In the case of the two unknown lens surface topographies, these 38 parameters are obtained through optimization. The input guess for the variables are those of the initial model for the conic ( $R$ and $Q)$ and all Zernike coefficients, $c_{n}=0$. In addition to these shape parameters, we consider that the lens, as a whole, will be probably displaced in both axes ( $\mathrm{x}_{0}$ and $\left.\mathrm{y}_{0}\right)$ and tilted /slanted (by angles $\alpha$ and $\beta$ ) from the nominal (initial) optical axis used to build the model, parallel to the keratometric axis. As we said before, we use the approximation that the keratometric and visual axes are nearly parallel (this would be true if the topographer were far enough from the eye; in practice the angle between these two axes would be below $0.5^{\circ}$ in most eyes).

Zernike coefficients alone (36 coefficients times 2 surfaces) provide more free parameters to adjust, than aberrometric data (usually 20 coefficients). Thus, they are highly powerful to fit topographies, but sometimes they are difficult to interpret, and they can even mask other important parameters such as tilts, etc. For this reason, we were interested in studying their effect upon the result of optimization. For this purpose, we have implemented an alternative simplified version, without Zernike polynomials, based on using biconic regular surfaces to 
describe the topographies of the lens surfaces. The canonical expression of the biconic is similar to the conic, but now we have two curvatures $\left(c_{x}=1 / R_{x}, c_{y}=1 / R_{y}\right)$ and two conic constants:

$$
z=\frac{c_{x} x^{2}+c_{y} y^{2}}{1+\sqrt{1-\left(1+Q_{x}\right) c_{x}{ }^{2} x^{2}-\left(1+Q_{y}\right) c_{y}{ }^{2} y^{2}}} .
$$

The optimization procedure is the same for both surface models (Eq. 1 or Eq. 2): The first stage is to choose a merit function to be minimized in the optimization process. For this purpose, we basically simulate the compensation of the wave aberration of the eye using phase plates ${ }^{41}$. Thus we introduce, into the model, a phase plate with the measured total WA, but with opposite sign, so that it should cancel that of the eye. In this simulation we can place the phase plate at the entrance pupil plane to have an exact compensation, whereas in the experiment that was not possible and the plate was placed in front of the eye. If we now perform the ray tracing computation, the WA of the compound eye + plate system will correspond to the initial prediction error (right column, central row in Fig.1). This error can even be of the same order of magnitude as the experimental WA. The optimization will consist of minimizing the RMS wave aberration of the eye + plate system. In this way, when the phase plate is removed after optimization, then the eye model alone must replicate the measured WA. In addition, optical design software, such as $\mathrm{CodeV}^{\mathrm{TM}}$, permits one to introduce additional constraints and weights to the variables in the merit function. Thus the merit function to minimize is the sum of a main term, the total RMS WA of the eye + phase plate (the prediction error), plus a series of weighted squared deviations of each free parameter with respect to its input guess value. Thus, each variable contributes more to increase the merit function as it deviates from the initial guess value. The amount of such increase depends on the weight. These weights were previously optimized for each variable (except for Zernike coefficients which were always unweighted) in such a way 
that they have a significant but not too strong contribution to the merit function. This is a method to avoid excessive (and potentially uncontrolled and unnecessary) deviations from the generic model. In optical design it is also important to give a strong weight to the focal length of the system, to keep the image in focus, and hence this is also included. The weights used in the merit function were $\mathrm{w}_{\mathrm{f}}=3$ for the focal length; $\mathrm{w}_{\mathrm{R}}=0.07$ for the curvature radii; $\mathrm{w}_{\mathrm{d}}=0.01$ for decentrations and tip/tilt angles, and $\mathrm{w}_{\mathrm{Q}}=0.0005$ for conic constants. Thus we have a hard constraint for the lens power and soft constraints for the lens geometry. As we discuss later, these values give an idea of the relative influence of the different parameters during the optimization. Curvature radii appear as the most critical parameters, while conic constants seem to have little influence.

The practical implementation was divided in three stages to have more control in the process:

1.- Introduce the phase plate at the pupil and adjust only the curvature radii $R_{a}$ and $R_{p}$ of the conic anterior and posterior lens conic surfaces to cancel defocus. The strategy applied here was to either keep constant or weight the initial ratio $-R_{a} / R_{p}$ instead of weighting each radius independently. The initial guess for this ratio is 1.7 for model \#1 or 1.53 for model \#2. When using the biconic surface we can also adjust astigmatism. The resulting radii are used as input guess in the next stage.

2.- The first attempt to reproduce the wave aberration is the optimization of the overall shape, position and orientation of the lens, minimizing the merit function. The free (but deviation-weighted) parameters are the radii and conic constants of both surfaces, and decentrations and tip/tilt angles of the whole lens as a solid. The input guess values for the conic constants are those of the generic model, zero for decentrations, and angles $\alpha=0^{\circ}$ and $\beta=5^{\circ}$. The 
procedure is equivalent for conic or biconic surfaces. At this stage we exclude Zernike coefficients, since preliminary results ${ }^{42}$ seemed to suggest that Zernike polynomials could convert each lens surface into a sort of phase plate able to modify the wavefront to reproduce the total WA. Consequently, the idea here is to study to what extent we can approximate the WA of the eye using a simpler regular surface (either conic or biconic) with a small number of parameters. In this way we also avoid an early phase plate potential effect. In the case of the biconic we stop the optimization here, as this surface model might, in theory, provide enough parameters to adjust some of the main aberration modes found in normal eyes (astigmatism, coma and spherical aberration).

3.- Fine tuning of the model by introducing and adjusting Zernike coefficient $c_{n}$ of the Zernike polynomials (up to 7th order) into the two previously adjusted conic surfaces. The overall shape and position of the lens is that found in the previous stage, which remains unchanged. The initial guess value is set to zero for all coefficients.

We want to point out that splitting the optimization into these three steps is not totally necessary, but it allows us to have some insight on this complex "black box" optimization process. In particular, splitting stages 2 and 3 is important to separate the fitting of the overall shape from the fine tuning.

\subsection{Experimental procedure}

Customized model eyes have been built for 19 eyes (12 subjects). 8 eyes corresponded to trained personnel in the clinic and experimenters, and 11 eyes were pre-LASIK surgery patients; 10 eyes corresponded to male and 9 to female subjects. In all cases eyes were normal and 
healthy; patients were passing preoperative exams for myopic refractive surgery. In most cases, data were collected under mild cycloplegia (2 drops of tropicamide 1\%). Only in 4 eyes of the trained group we used full cycloplegia (2 drops of cyclopentolate 1\%). Ages ranged from 23 to 46 years, mean $32 \pm 8$ years. We performed both anatomical and optical measurements. The elevation topography of the corneal anterior surface was measured with an ORBSCAN $\mathrm{II}^{\mathrm{TM}}$ (Bausch \& Lomb) topographer. This also provides the pupil position and axis, which is essential to build the customized model. For each patient we obtained 3 topographies and computed the average. The axial thickness of the optical media was measured by ultrasound biometry (OCUSCAN $^{\mathrm{TM}}$, Alcon). In this case at least 5 measurements were taken and averaged (discarding outliers). The average thicknesses obtained were $0.55 \pm 0.02 \mathrm{~mm}$ for the cornea, 3.47 $\pm 0.28 \mathrm{~mm}$ for the aqueous, $3.99 \pm 0.26 \mathrm{~mm}$ for the lens and $16.62 \pm 0.92 \mathrm{~mm}$ for the vitreous The mean axial length was $24.09 \pm 0.86 \mathrm{~mm}$. These mean values are close to those of the generic models which represent classic values. The analysis of the topographies gave the following data: average vertex radius $7.59 \pm 0.27 \mathrm{~mm}$, conic constant $-0.33 \pm 0.12$; RMS conic fit error 11.56 micrometers. This corneal radius is lower than classic values, which would be one of the potential sources for the average myopia measured in this group of patients (see below). The average pupil center was displaced from the corneal vertex $0.14 \pm 0.22 \mathrm{~mm}$ and $0.004 \pm 0.17 \mathrm{~mm}$ in $\mathrm{X}$ and $\mathrm{Y}$ directions respectively.

Optical measurements with ZYWAVE ${ }^{\mathrm{TM}}$ (Bausch \& Lomb) provided second and higher order total aberrations. Other additional data were taken, visual acuity, subjective refraction, objective manifest refraction, (autorefractometer KR8100 ${ }^{\mathrm{TM}}$, Topcon) but were not used directly to build the customized eye models. Wavefront refraction provided by the aberrometer was usually slightly different from that provided by the autorefractometer. The later gave spheres 
from $-8 \mathrm{D}$ to $+0.41 \mathrm{D}$, average $-2.75 \pm 2.52 \mathrm{D}$; compared to wavefront values $-8.1 \mathrm{D}$ to +0.41 $\mathrm{D}$, average $-2.98 \pm 2.63 \mathrm{D}$. In 8 eyes the differences between the two instruments were less than $0.25 \mathrm{D}$, and only in 3 cases the difference was greater than $0.75 \mathrm{D}$. Autorefractometer cylinder refraction ranged from $-2 \mathrm{D}$ to $-0.12 \mathrm{D}$, average $-0.66 \pm 0.44 \mathrm{D}$; and wavefront astigmatism from $-1.9 \mathrm{D}$ to $-0.14 \mathrm{D}$, average $-0.58 \pm 0.44 \mathrm{D}$; mean axis $88^{\circ} \pm 68^{\circ}$. Thus, both types of measurements provide consistent results in most cases. In what follows, we use the wavefront data, since the goal in the optimization of the model is to reproduce the measured wavefront. The pupil diameter varied from subject to subject, always being greater than $6 \mathrm{~mm}$; this was the standard diameter used in all cases.

\section{RESULTS}

The results regarding the prediction of aberrations are given in Figure 2 for 19 eyes and the two models attempted. Fig.2 displays RMS prediction errors, in micrometers, for the different stages of the customization process. These values correspond to the total WA including second and higher order aberrations. As a reference, the measured RMS WA (not included in these plots) ranged from 0.6 to 11.92 micrometers, average $4.5 \pm 3.36$ micrometers (about 8 wavelengths), which is the sum of second order ( $-3 \mathrm{D}$ average defocus and $-0.58 \mathrm{D}$ average astigmatism) and higher order aberrations. This total aberration was the target that we want to reproduce by the customized eye model. 
One interesting result is that upper and lower panels are quite similar. This means that both initial models perform equally in predicting the measured WA. For each eye, the four bars tend to be progressively lower, as they correspond to more advanced stages in the customization process. The Initial state represents the prediction error resulting after the first stage, consisting of replacing generic by patient data into the model. We can see that this stage permits a first crude approximation to the target value, with an average prediction error of nearly 1 micrometer which is of the order of the $20 \%$ of the average wave aberration measured. In the second stage, optimization permits us to substantially improve this error. However, the initial result obtained when we consider conic lens surfaces is rather modest, as the error only decays from 1 to about 0.8 micrometers on average. In other words, with the conic surfaces we can basically reproduce the defocus term, but we can not adjust astigmatism and other higher order aberrations (trefoil, etc.) In fact, when we introduce biconics, the prediction error reduces quite significantly, by a factor of two at least, being now lower than the wavelength in most cases; average 0.36 and 0.39 $\mu \mathrm{m}$ for models \#1 and \#2 respectively. Finally, when we introduce the Zernike polynomials, we obtain an improvement of two orders of magnitude. In fact, the last bars, corresponding to the final conic plus Zernikes surfaces are barely visible, indicating remarkably small prediction errors in most cases. For model \#1, the final RMS errors range from 0.0013 to $0.018 \mu \mathrm{m}$; average $0.0066 \mu \mathrm{m}$. Results are similar for model \#2, with a somewhat higher variability, range 0.00075 to $0.032 \mu \mathrm{m}$; average $0.0049 \mu \mathrm{m}$. These prediction errors are always lower than experimental variability, and about one order of magnitude on average, as measurement errors are typically around 0.05 micrometers for a $6 \mathrm{~mm}$ pupil ${ }^{43}$. As we said above, a remarkable aspect is that no significant differences appear between the prediction errors provided by the two different models (constant or gradient index lens). In both cases we obtained a $100 \%$ success in predicting the 
measured optical quality. The prediction error is extremely low (average about $\lambda / 100$ ) and the convergence of the optimization procedure seems guaranteed in all cases attempted so far.

\section{\#\#\#\#\#\#\#\#\#\#\#\# Insert Figure 2 about here \#\#\#\#\#\#\#\#\#\#}

Two examples of predicted and measured higher order aberrations are given in Figures 3 and 4. JOR, eye \#3 (Fig. 3) was taken among those having highest final errors, whereas AMD, eye \#10 (Fig. 4) represents one of the best cases, with low prediction errors. In both Figures, the upper panel represents the measured higher order wave aberration (HOA), the second row corresponds to the HOA of the model, when we depart from the generic model \#1 and the bottom row to the results obtained departing from model \#2. We have chosen to represent HOA, instead of including second order contribution, which is usual in ocular aberrometry. In addition, these maps are quite similar to the total aberration, except for the conic surfaces, where astigmatism can take significant values. In Fig. 4 (AMD) we can see that even the conic surfaces (left panels) provide a good visual resemblance with the experimental data. This visual resemblance further improves with the biconic surfaces, and is almost identical to the original when we introduce the Zernike polynomials. The two models give almost the same results. However, this visual resemblance can be somewhat misleading: Visually, the change between biconic and Zernikes surfaces seems small, but the RMS difference changes by 2 orders of magnitude. Subject JOR displays a somewhat different behavior. Now, conic and biconic surfaces provide a quite poor visual resemblance with experimental data, and only after optimization of Zernike coefficients do we obtain a good result. (Note that the large difference between conic and biconic cases is mainly due to astigmatism, which is not included in the HOA representation.) In summary, the 
optimization stage was totally necessary to obtain an average prediction error lower than 1 micrometer. The use of conic surfaces to model the lens does not provide satisfactory results. The biconic surfaces often provide a good visual resemblance with the experimental wavefront, but the RMS prediction error is still high in most cases (average about 0.36 micrometers or more). However, the complete optimization process, considering conic plus Zernike polynomials surfaces always provided a satisfactory prediction of the total wave aberration.

\#\#\#\#\#\#\#\#\#\#\# Insert Figures 3 and 4 about here \#\#\#\#\#\#\#\#\#\#

\section{DISCUSSION}

So far we have presented a method to develop personalized eye models, departing from any generic average model (here he have compared two totally different models, \#1 and \#2), replacing generic values by available individual data, and then performing a 3-steps optimization. The method has been shown to be highly efficient and robust (100\% success) for the 19 eyes tested, to achieve the goal of reproducing the measured total wave aberration of each eye, with a high fidelity. Furthermore, the resulting wavefront prediction was basically independent of the initial generic model, no matter whether it considers either a constant or a gradient index lens. We consider that this is a significant step towards the development of accurate models of individual eyes, as the main goal of predicting the measured optical quality has been demonstrated. As far as we know, such high fidelity reproductions of the measured wavefront have not been reported before. Nevertheless, eye models must be both anatomically

and optically accurate. In a preliminary study ${ }^{42}$ we found that, in general the lens optimization 
with no additional constraints could yield several different solutions (lack of uniqueness). For this reason, we have developed this 3-steps method, with constraints on the variables. In this way, we can constrict the space of possible solutions and choose the solution closer to the initial generic model, which is mainly constituted of averages of anatomical data. This is accomplished by keeping the ratio between the anterior and posterior radii constant in the fist step, and by including a soft constraint to the variables in the second step. These constraints are implemented by incorporating a penalty proportional to the deviation of each variable from its initial generic value into the merit function. This strategy has been highly successful, guaranteeing a unique solution and convergence. However, as we discuss next, if we depart from different initial generic models, then we arrive at different geometries of the optimized lens, as one may expect from the fact that the two initial models consider totally different refractive index distributions. Therefore, the resulting models are highly precise optically, but the issue of their anatomical accuracy requires further analysis.

\section{Lens topography}

The geometrical parameters describing the resulting lens topographies are summarized in Table 2 for both models. For each variable, we include average, standard deviation and range (minimum and maximum values within brackets). The upper rows correspond to the anterior lens surface, the middle rows to the posterior surface, and the lower row to global data of the lens as a solid (position, orientation and power). The surface topographies are those obtained at the final stage, defined by a revolution conic plus a Zernike polynomials expansion. The Table includes 3 parameters for each surface, the radius, conic constant, and the RMS sagitta of the Zernike expansion in micrometers. The last parameter is a measure of the difference between the conic 
and the final topography, or in other words, the conic deformation introduced by the last optimization stage. Figure 5 illustrates the individual variability for the particular case of the curvature radii, which is the most important parameter. The two panels (anterior and posterior surfaces respectively) include the initial radii of the generic models \#1 and \#2 (dashed and continuous straight lines respectively) as a reference. We can see that, the general trend is that the optimized radii tend to be closer to their respective initial values. However, model \#1 (constant refractive index) yields lower radii for the anterior surface, which seems to be compensated by the posterior surface, for which we obtain more negative values (higher magnitude). In other words, the constant refractive index model shows a tendency to equalize the refractive power of both lens surfaces. Another feature of this model is to provide a lower intersubject variability. On the contrary, the GRIN lens model shows a much larger variability, it keeps the posterior radius closer to the initial value (the mean is basically equal to the generic model), and yields (in several cases) anterior radii substantially higher than the initial value. In fact, various optimized eye models \#2 show a quite flat anterior surface.

\section{\#\#\#\#\#\#\#\#\#\#\#\# Insert Table 2 about here \#\#\#\#\#\#\#\#\#\# \#\#\#\#\#\#\#\#\#\#\#\# Insert Fig. 5 about here \#\#\#\#\#\#\#\#\#\#}

These results can be largely explained by the different refractive index distributions of the two models. First of all, we have to bear in mind that most of these eyes are myopic (average $-3 \mathrm{D}$ ). The average resulting power of the optimized lens is greater than $23 \mathrm{D}$ (see Table 2) and is about the same for most eyes, except for a couple of exceptions, with significantly higher powers, up to 28 D. These resulting powers are higher than those of the initial models (21.8 D for model \#1 
and 22.1 D for model \#2). For the constant refractive index lens we have an average increment of $1.2 \mathrm{D}$ in the lens power to fit the refractive state. Roughly speaking, this average excess in lens power would explain about one third of the average observed myopia. For a constant refractive index lens, the most efficient way to increase its power is to have a more curved anterior surface, which is what we obtain through optimization. For the GRIN model, the increment in power is smaller (0.8 D), but here the trend seems to be opposite. Even for myopic subjects, the anterior surface is flatter than standard anatomical values. One plausible explanation is that the GRIN lens model used is not adaptive, but it was optimized for a given lens geometry ${ }^{21}$. That is, we can adjust the surface geometry, but the internal refractive index distribution remains constant, and then it does not adapt to the optimized surface topography. In our current implementation we have developed only one first stage, consisting of adapting the GRIN model to the measured lens thickness, by stretching or shrinking the distribution along the optical axis. The lack of adaptation seems to produce a sort of excess of refractive power in some eyes, so that the anterior surface has to be necessarily flatter in order to fit the global refractive state of the eye. In summary, when we compare the resulting radii with standard experimental values, models \#1 and \#2 seem to underestimate or overestimate respectively the radius of the anterior lens surface. The most plausible explanation is that none of the two refractive index models used correspond to the actual GRIN distribution of the modeled eyes. In addition, these results could indicate that the actual refractive index distribution is neither constant nor quadratic (model \#2). Furthermore, a recent study ${ }^{39}$ suggests that there could be an important intersubject variability in the refractive index distribution within the lens, and provides solid evidence for a continuous change with age. Therefore, one of the most important open problems in obtaining anatomically accurate custom models is the need to have an adaptive index distribution model which can adapt to the surface 
topography, dimensions, age of the lens, and even accommodation. Consequently, this will be the subject of further work.

The resulting conic constants are similar in both models, but they clearly have more negative values than those of the generic models. Decentrations are small (a few tenths of milimiter) and the average orientations are basically equal to the initial values, $\alpha=0$ and $\beta=5^{\circ}$ (we adopted the value of model $\# 2{ }^{21}$ also for model \#1). As we discuss below, this seems to indicate that these parameters have a relatively low influence on the wave aberration, so that even the small weights used for these variables in the merit function were enough to maintain their initial values unchanged during optimization. Finally, the column corresponding to the RMS Zernike coefficients indicates the amount of deformation that we have to introduce in the conic surfaces to reproduce the total wavefront of the eye. We can see that the average RMS deformation, or average distance from the final topography to the conic surface is similar in both models, ranging from 50 to 75 micrometers. As a reference, we can compare these values to corneal topography data. In a recent study on 127 eyes $^{44}$, the average RMS deformation between the topography and the best fit conic surface found was about 18 micrometers. Roughly speaking, this means that our optimization procedure provides 3 times greater deformations (with respect to the conic) for the lens topography than those found in the cornea. It is difficult to tell whether these values can be anatomically plausible, as a direct comparison is not possible because there is a lack of experimental data on this matter. Nevertheless, some theoretical studies on lens topography ${ }^{45}$ suggest that the conic model is not adequate, as it can only fit the lens topography near the poles (paraxial zone), and one has to consider a progressive deformation towards the equator to fit the lens shape. Consequently, the order of magnitude that we find through optimization could be 
plausible in anatomical terms. Nevertheless, this is a rather new and open question, which also deserves further work, both experimental and theoretical.

\section{Tolerance analysis}

The proposed method bears intrinsic uncertainties in the sense that the whole lens geometry is unknown initially. The geometry is then adjusted to fit the observed (measured) optical performance, with the sole constraint that the result is as close as possible to the initial generic model. This is basically an optical design problem, where it is essential to perform a tolerance analysis to determine how critical the optimised values for the different variables are for the prediction of the total wave aberration of the eye. We have developed the following procedure for this particular optical design problem: Once the optimization algorithm finds the minimum of the merit function, we obtain one-dimensional plots of this merit function versus each variable around the optimal (minimum) value. One example is given in Fig. 6 for the case of Fig. 4, model \#1, biconic surfaces. We have chosen this since here the biconic surfaces provided a reasonably good prediction of the wave aberration. We avoided performing this type of analysis with the 36x2 Zernike coefficients, which would be tedious and not particularly significant at this stage. From these plots, we can see that the curvature radii are indeed the most critical parameters, especially for the posterior surface, which shows the narrowest minimum of the merit function. On the opposite side, surprisingly, the conic constants show highly flat and wide minima, indicating that conic constants of the lens surfaces (especially the anterior Q) have little impact on the merit function, and hence on the total wave aberration of the eye. Nevertheless, there seems to be some correlation between the tolerances for the radius and conic constants. Both parameters are more critical for the posterior surface as compared to the anterior ones. 
Another interesting finding is that the orientation angles of the lens are barely critical. This explains why the final optimised values are the same as the initial ones. In comparison, decentrations seem more critical parameters, but if we consider their values (tenths of mm), they are not especially critical in relative terms.

\section{\#\#\#\#\#\#\#\#\#\#\#\# Insert Fig. 6 about here \#\#\#\#\#\#\#\#\#\#}

We can extract more information for these plots. A typical uncertainty measure for a peak is its full width at half height (FWHH) or half of this value. A natural adaptation of this definition for a minimum is its FW at double height (FWDH). Applying this measure $( \pm \mathrm{FWDH} / 2)$ for this particular case (and this result can be extrapolated to other cases to a first approximation), we obtain a precision of $\pm 0.5 \mathrm{~mm}$ for the anterior radii; $\pm 0.2 \mathrm{~mm}$ for the posterior radii; \pm 1.8 and \pm 0.7 for the anterior and posterior conic constants respectively; $\pm 0.45 \mathrm{~mm}$ and $\pm 0.7 \mathrm{~mm}$ for the $\mathrm{X}$ and $\mathrm{Y}$ decentrations; and $\pm 2.6^{\circ}$ for both orientation angles $\alpha$ and $\beta$. These values provide an indication of the maximum deviations of the variables from their optimal values, which guarantee that the merit function is close to its minimum, and hence the prediction error of the wave aberration of the eye can be tolerated. In relative terms, the curvature radii are the most critical parameters with tolerances about 5\% and 3\% respectively, as they determine the refractive power of the lens. Other parameters with a potential impact on higher order aberrations have much higher relative tolerances, about 35\% for conic constants, etc. Therefore, it is possible to analyze the uncertainty and accuracy of the geometrical parameters obtained through optimization. 
In summary, we have proposed, developed and analyzed a method to build custom optical models of individual eyes. The results are highly satisfactory and the proposed method seems highly promising. The method guarantees the first of the two main goals for an eye model, namely to be optically accurate. For the geometry of the lens there are still several open questions which require further work. The results obtained are compatible with the refractive index models used, but the two models used gave underestimation or overestimation, respectively, of the initial curvature radii, indicating that the real anatomical distribution must be something in between. Another essential aspect is experimental validation. There have been important advances towards a reliable in vivo measurement of the lens topography, and techniques such as OCT seem highly promising, but this problem also needs further research. Therefore, we believe that the present study represents a decisive step, but further work and developments are required to achieve the goal of having models which reproduce with high fidelity both the anatomy and optical properties of the eye under study. Apart from experimental techniques for the measurement of lens topography, and other additional optical performance measurements, we believe that the optical optimization tools will also be essential, as well as better models of the refractive index distribution in the lens.

\section{Acknowledgements}

This research has been partially supported by the Spanish CICyT, grant FIS2005-05020-C03-01 and by the clinic RealVision. 


\section{References}

1.- S. Klein, "Optimal corneal ablation for eyes with arbitrary Hartmann-Shack aberrations", J. Opt. Soc. Am. A 15, 2580-2588 (1998).

2.- S. MacRae, J. Schwiegerling, and R. W. Snyder, "Customized and low spherical aberration corneal ablation design", J. Refract. Surg. 15, S246-S248 (1999).

3.- M. Mrochen, C. Donitzky, C. Wullner, and J. Loffler, "Wavefront-optimized ablation profiles: Theoretical background", J. Cataract Refract. Surg. 30, 775-785 (2004).

4.- P.-R. Preussner, J. Wahl, and D. Weitzel, "Topography-based intraocular lens power selection", J. Cataract Refract. Surg. 31, 525-533 (2005).

5.- D. Atchison and G. Smith, Optics of the Human Eye, Butterworth-Heinemann (2000).

6.- A. Gullstrand, “The Optical System of the Eye”. In: Handbuch der Physiologische Optik, 3rd edn, Vol. 1, ed. H. von Helmholtz.(Voss, Hamburg 1909), 350-358.

7.- Y. Le Grand and S. G. El Hage, Physiological Optics (Springer-Verlag, New York, 1980), pp. 54-55.

8.- L. N. Thibos, M. Ye, X. Zhang, and A. Bradley, “The chromatic eye: a new reduced-eye model of ocular chromatic aberration in humans,’’ Appl. Opt. 31, 3594-3600 (1992).

9 .- J. W. Blaker, “'Toward an adaptive model of the human eye,’ J. Opt. Soc. Am. 70, 220-223 (1980).

10.- O. Pomerantzeff, M. Pankratov, G.-J. Wang, and P. Dufault, “Wide-angle optical model of the eye,’ Am. J. Optom. Physiol. Opt. 61, 166-176 (1984).

11.- M. C. W. Campbell, "Measurement of refractive index in an intact crystalline lens,'” Vision Res. 24, 409-415 (1984). 
12.- B. K. Pierscionek and D. Y. C. Chan, “Refractive index gradient of human lenses,” Optom. Vis. Sci. 66, 822-829 (1989).

13.- G. Smith and D. A. Atchison, "The gradient index and spherical aberration of the lens of the human eye", Ophthal. Physiol. Opt. 21, 317-326 (2001).

14.- W. Lotmar, “Theoretical eye model with aspherics,’’ J. Opt. Soc. Am. 61, 1522-1529 (1971).

15.- R. Navarro, J. Santamaría, and J. Bescós, “Accommodation dependent model of the human eye with aspherics,’’ J. Opt. Soc. Am. A 2, 1273-1281 (1985).

16.- A. C. Kooijman, “'Light distribution on the retina of a wideangle theoretical eye,’ J. Opt. Soc. Am. 73, 1544-1550 (1983).

17.- I. Escudero-Sanz and R. Navarro, "Off-axis aberrations of a wide-angle schematic eye model", J. Opt. Soc. Am. A, 16, 1-11 (1999).

18 - S. Norrby, "The Dubbelman eye model analysed by ray tracing through aspheric surfaces", Ophthal. Physiol. Opt. 25, 153-161 (2005).

19.- J. Schwiegerling, J. E. Greivenkamp, J. M. Miller et al., " Optical modeling of radial keratotomy incision patterns.", Am. J. Ophthalmol. 122, 808-817 (1996).

20.- D. Siedlecki, H. T. Kasprzak, B. K. Pierscionek, "Schematic eye with a gradient-index lens and aspheric surfaces,” Opt. Lett. 29, 1197-1199 (2004).

21.- H.-L. Liou and N. A. Brennan, “Anatomically accurate, finite model eye for optical modeling,’' J. Opt. Soc. Am. A 14, 1684-1695 (1997).

22.- H. C. Howland and B. Howland, "A subjective method for the measurement of the monochromatic aberrations of the eye", J. Opt. Soc. Am. A, 67, 1508-1518 (1977). 
23.- J. Liang and D. R. Williams, "Aberrations and retinal image quality of the normal human eye", J. Opt. Soc. Am. A 14, 2873-2883 (1997).

24.- R. Navarro, E. Moreno, and C. Dorronsoro, "Monochromatic aberrations and point-spread functions of the human eye across the visual field", J. Opt. Soc. Am. A 15, 2522-2529 (1998). 25.- A. Guirao and P. Artal, "Corneal wave aberration from videokeratography: accuracy and limitations of the procedure", J. Opt. Soc. Am. A, 17, ,955-965 (2000).

26.- S. Barbero, S. Marcos, J. Merayo-Lloves, and E. Moreno-Barriuso, "Validation of the estimation of corneal aberrations from videokeratography: A test on keratoconus eyes", J. Cataract. Refract. Surg. 28, 1594-1603 (2002).

27.- M. Millodot and J. Sivak, "Contribution of the cornea and lens to the spherical aberration of the eye", Vision Res. 19, 685-687 (1979).

28.- P. Artal, A. Guirao, E. Berrio, D. R. Williams, "Compensation of corneal aberrations by internal optics in the human eye", J. Vision, 1 , 1-8 (2001).

29.- M. Dubbelman, H. A.Weeber, G. L. van der Heijde and H. J. Völker-Dieben, "Radius and asphericity of the posterior corneal surface determined by corrected Scheimpflug Photography,” Acta Ophthalmol. Scand. 80, 379-383 (2002).

30.- T. Kirschkamp, M. Dunne and J. C. Barry, "Phakometric measurement of ocular surface radii of curvature, axial separations and alignment in relaxed and accommodated human eyes,” Ophthal. Physiol. Opt. 24, 65-73 (2004).

31.- J. F. Koretz, C. A. Cook, P. L. Kaufman “Aging of the human lens: changes in lens shape at zero-diopter accommodation,” J. Opt. Soc. Am. A 18, 265-272 (2001).

32.- M. Dubbelman, G. L. van der Heijde, and H. A.Weeber, "Change in shape of the aging human crystalline lens with accommodation,” Vision Res. 45, 117-132 (2005). 
33.- J. F. Koretz , S. A. Strenk, L. M. Strenk, J. L. Semmlow, “Scheimpflug and high-resolution magnetic resonance imaging of the anterior segment: a comparative study”, J. Opt. Soc. Am. A, 21, 346-354 (2004).

34.- M. Dubbelman, R. G. L. van der Heijde, and H. A. Weeber, "Comment on "Scheimpflug and high-resolution magnetic resonance imaging of the anterior segment: a comparative study"', J. Opt. Soc. Am. A, 22, 1216-1218 (2005).

35.- S. Radhakrishnan, A. M. Rollins, J. E. Roth, and S. Yazdanfa, "Real-time optical coherence tomography of the anterior segment at $1310 \mathrm{~nm} "$, Arch. Ophthalmol. 119, 1179-1185 (2001). 36. - M. J. Howcroft and J. A. Parker, "Aspheric curvatures for the human lens'", Vision Res. 17, 1217-1223 (1977).

37.- P. M. Kiely, G. Smith, and L. G. Carney, "The mean shape of the human cornea", Optica Acta 29, 1027-1040 (1982).

38.- N. Brown, "The change in lens curvature with age", Exp. Eye Res. 19, 175-183 (1974). 39.- C. E. Jones, D. A. Atchison, R. Meder, and J. M. Pope, "Refractive index distribution and optical properties of the isolated human lens measured using magnetic resonance imaging (MRI)", Vision Res. 45, 2352-2366 (2005).

40.- J. Schwiegerling, J. Greivenkamp, and J. Miller, "Representation of videokeratoscopic height data with Zernike polynomials", J. Opt. Soc. Am. A. 12, 2105-2113 (1995).

41.- R. Navarro, E. Moreno-Barriuso, S. Bará, and T. Mancebo, "Phase-plates for waveaberration compensation in the human eye", Opt. Lett. 25, 236 - 238 (2000).

42.- R. Navarro, L. González, J. L. Hernández, "Prediction of optical aberrations by personalized eye models”, II Physiological Optics Topical Meeting of the European Optical Society, Granada, Spain (September 2004). 
43.- P. Rodriguez, R. Navarro, J. Arines, and S. Bará, "A calibration set for ocular aberrometers: Manufacture, testing and application", J. Refrac. Surgery, 11, xx-xx (2005).

44.- R. Navarro, R. González, and J. L. Hernández, "Optics of the average normal cornea from general and canonical representations of its surface topography", JOSA A 23, xxx-xxx (2006). 45.- H. T. Kasprzak, "New approximation for the whole profile of the human crystalline lens", Ophthal. Physiol. Opt. 20, 31-43 (2000). 
Table 1. The two generic eye models used in the customization procedure. The main difference is that model \#1 has a constant refractive index lens, whereas model \#2 assumes a quadratic distribution.

\begin{tabular}{lccccc}
\hline $\begin{array}{l}\text { Eye model \#1 } \\
\text { (Navarro et al. }{ }^{15} \text { ) }\end{array}$ & Type & $\begin{array}{c}\text { Radius } \\
\mathbf{( m m )}\end{array}$ & $\begin{array}{c}\text { Conic } \\
\text { constant }\end{array}$ & $\begin{array}{c}\text { Thickness } \\
(\mathbf{m m})\end{array}$ & $\begin{array}{c}\text { Refractive } \\
\text { index (nd }\end{array}$ \\
\hline Surface \#1 Cornea & conic & 7.72 & -0.26 & 0.55 & 1.376 \\
\#2 Aqueous & sphere & 6.5 & 0 & 3.05 & 1.3374 \\
\#3 Aqueous (stop) & plane & Infinity & 0 & 0 & 1.3374 \\
\#4 Lens & conic & 10.2 & -3.13 & 4 & 1.42 \\
\#5 Vitreous & conic & -6 & -1 & 16.32 & 1.336 \\
\hline
\end{tabular}

\begin{tabular}{|c|c|c|c|c|c|}
\hline $\begin{array}{l}\text { Eye model \#2 } \\
\left(\text { Liou \& Brennan }^{21}\right)\end{array}$ & Type & $\begin{array}{l}\text { Radius } \\
\text { (mm) }\end{array}$ & $\begin{array}{c}\text { Conic } \\
\text { constant }\end{array}$ & $\begin{array}{c}\text { Thickness } \\
\text { (mm) }\end{array}$ & $\begin{array}{l}\text { Refractive } \\
\text { index }\left(\mathbf{n}_{d}\right)\end{array}$ \\
\hline Surface \#1 Cornea & conic & 7.77 & -0.18 & 0.5 & 1.376 \\
\hline \#2 Aqueous & conic & 6.4 & -0.6 & 3.16 & 1.336 \\
\hline $\begin{array}{l}\text { \#3 Anterior lens } \\
\text { (stop) }\end{array}$ & conic & 12.4 & -0.94 & 1.59 & GRIN A \\
\hline \#4 Posterior lens & plane & Infinity & 0 & 2.43 & GRIN P \\
\hline \#5 Vitreous & conic & -8.1 & 0.96 & 16.27 & 1.336 \\
\hline $\begin{array}{l}\text { GRIN } \\
\text { Parameters }\end{array}$ & $\mathbf{n}_{00}$ & $\mathbf{n}_{01}$ & $\mathbf{n}_{02}$ & $\mathbf{n}_{10}$ & \\
\hline Anterior & 1.368 & 0.049057 & -0.015427 & -0.001978 & \\
\hline Posterior & 1.407 & 0.00 & -0.006605 & -0.001978 & \\
\hline
\end{tabular}


Table 2. Topography data of the lens surfaces resulting after optimization. Upper values are average \pm standard deviation over 19 eyes; lower values indicate range [minimum, maximum].

\begin{tabular}{|c|c|c|c|c|c|c|}
\hline \multicolumn{7}{|c|}{ Anterior lens surface } \\
\hline & \multicolumn{2}{|c|}{ Radius (mm) } & \multicolumn{2}{|c|}{ Conic constant } & \multicolumn{2}{|c|}{$\begin{array}{l}\text { RMS Zernikes } \\
\text { (micrometers) }\end{array}$} \\
\hline Model \#1 & \multicolumn{2}{|c|}{$7.74 \pm 1.1$} & \multicolumn{2}{|c|}{$-4.7 \pm 2.7$} & \multicolumn{2}{|c|}{$51.6 \pm 19.9$} \\
\hline Model \#2 & \multicolumn{2}{|c|}{$\begin{array}{c}14.6 \pm 7 \\
{[7.2,31.3]}\end{array}$} & \multicolumn{2}{|c|}{$\begin{array}{c}-5 \pm 1.8 \\
{[-8.7,-2.65]}\end{array}$} & \multicolumn{2}{|c|}{$\begin{array}{l}62.8 \pm 20.6 \\
{[12.7,92.7]}\end{array}$} \\
\hline \multicolumn{7}{|c|}{ Posterior lens surface } \\
\hline & \multicolumn{2}{|c|}{ Radius (mm) } & \multicolumn{2}{|c|}{ Conic constant } & \multicolumn{2}{|c|}{$\begin{array}{l}\text { RMS Zernikes } \\
\text { (micrometers) }\end{array}$} \\
\hline Model \#1 & \multicolumn{2}{|c|}{$\begin{array}{c}-6.7 \pm 0.9 \\
{[-8.35,-5.2]}\end{array}$} & \multicolumn{2}{|c|}{$\begin{array}{c}-1.6 \pm 2.6 \\
{[-6.1,2.36]}\end{array}$} & \multicolumn{2}{|c|}{$\begin{array}{c}74.3 \pm 28 \\
{[42.5,126.6]}\end{array}$} \\
\hline Model \#2 & \multicolumn{2}{|c|}{$\begin{array}{l}-8.1 \pm 1.45 \\
{[-10.4,-5.5]}\end{array}$} & \multicolumn{2}{|c|}{$\begin{array}{c}-2.5 \pm 4.3 \\
{[-10.3,4.8]}\end{array}$} & \multicolumn{2}{|c|}{$\begin{array}{c}53.3 \pm 30.8 \\
{[18.1,158]}\end{array}$} \\
\hline \multicolumn{7}{|c|}{ Position, orientation \& power } \\
\hline & $\alpha$ (deg.) & $\beta$ (deg.) & $\Delta \mathbf{x}(\mathbf{m m})$ & & & $\begin{array}{c}\text { Power } \\
\text { (diopters) }\end{array}$ \\
\hline Model \#1 & $\begin{array}{c}0 \pm 0.03 \\
{[-0.08,0.04]}\end{array}$ & $\begin{array}{l}5.01 \pm 0.02 \\
{[4.99,5.06]}\end{array}$ & $\begin{array}{c}0.15 \pm 0.23 \\
{[-0.54,0.42]}\end{array}$ & $\begin{array}{l}0.1 \\
{[-0}\end{array}$ & $\begin{array}{l}.23 \\
.39]\end{array}$ & $\begin{array}{r}23.2 \pm 1.6 \\
{[21.1,28.2]}\end{array}$ \\
\hline Model \#2 & $\begin{array}{l}-0.01 \pm 0.04 \\
{[-0.05,0.04]}\end{array}$ & $\begin{array}{c}5 \pm 0.05 \\
{[4.93,5.15]}\end{array}$ & $\begin{array}{c}0.37 \pm 0.19 \\
{[-0.15,0.67]}\end{array}$ & $\begin{array}{l}-0 . \\
{[-0}\end{array}$ & $\begin{array}{r}0.29 \\
.33]\end{array}$ & $\begin{array}{c}23.5 \pm 1.7 \\
{[21.2,27.9]}\end{array}$ \\
\hline
\end{tabular}




\section{FIGURE CAPTIONS}

Fig. 1. Schematic diagram of the customization method.

Fig. 2. WA prediction errors (RMS) for the different optimization stages. The two initial generic models (upper and lower panels) provide equivalent results.

Fig. 3. Experimental (upper panel) and predicted wave aberration for subject JOR (eye \#3). From left to right we can see a progressive improvement when using more complete surface models. Model \#1 considers constant refractive index lens and model \#2 a quadratic GRIN lens.

Fig. 4. Experimental (upper panel) and predicted wave aberration for subject AMD (eye \#10).

Fig. 5. Curvature radii of the anterior and posterior lens surfaces obtained for 19 eyes and two (\#1 and \#2) models. Straight lines (dashed and continuous) correspond to the initial values of the two generic models respectively.

Fig. 6. Tolerance analysis for subject AMD, model \#1, biconic surfaces. The plots represent onedimensional slices of the merit function around its minimum, along the different variables. 


\section{Patient Data $\quad$ Optical Model Prediction Error}

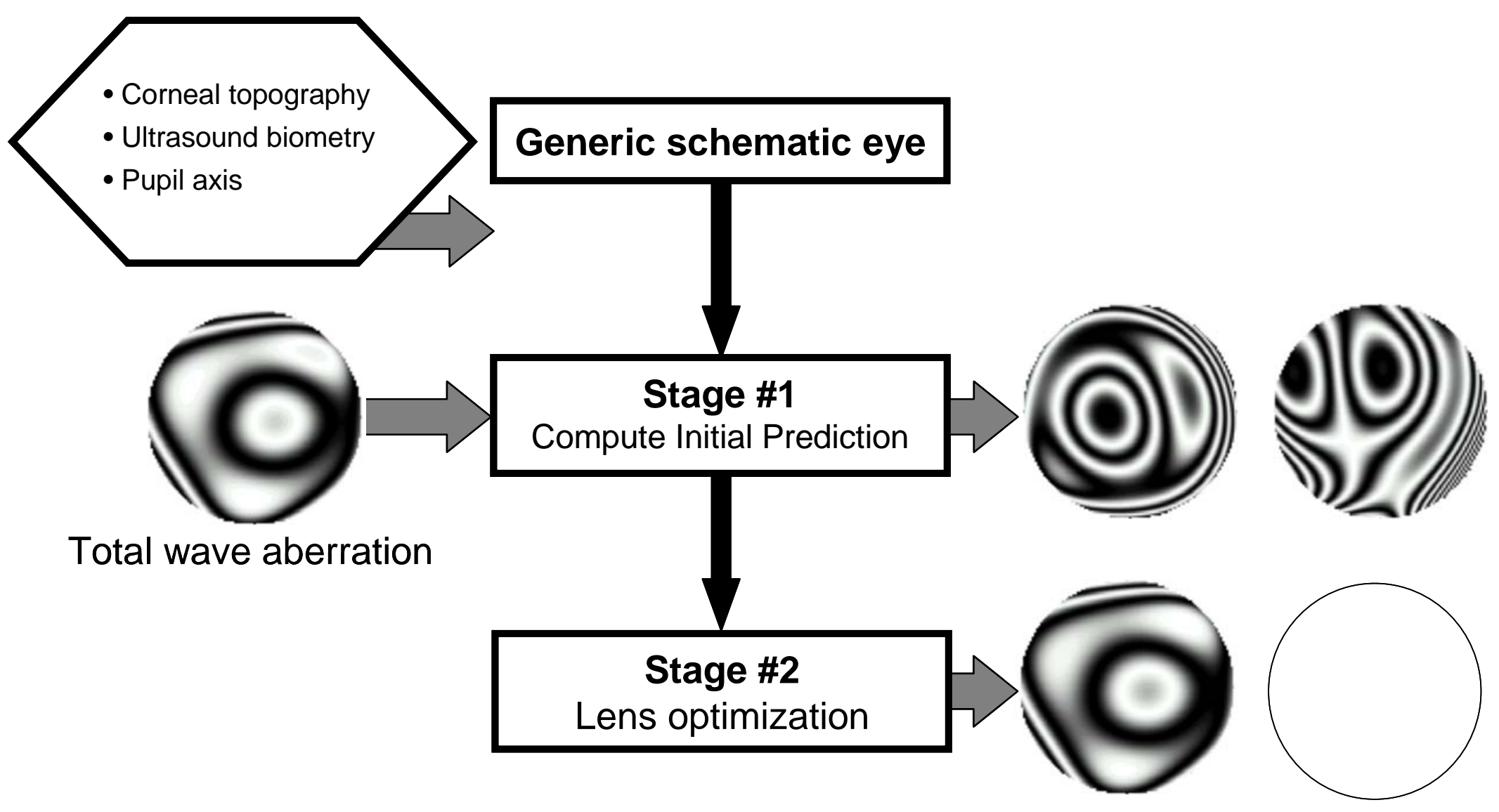

FIG. 1 
Model \#1

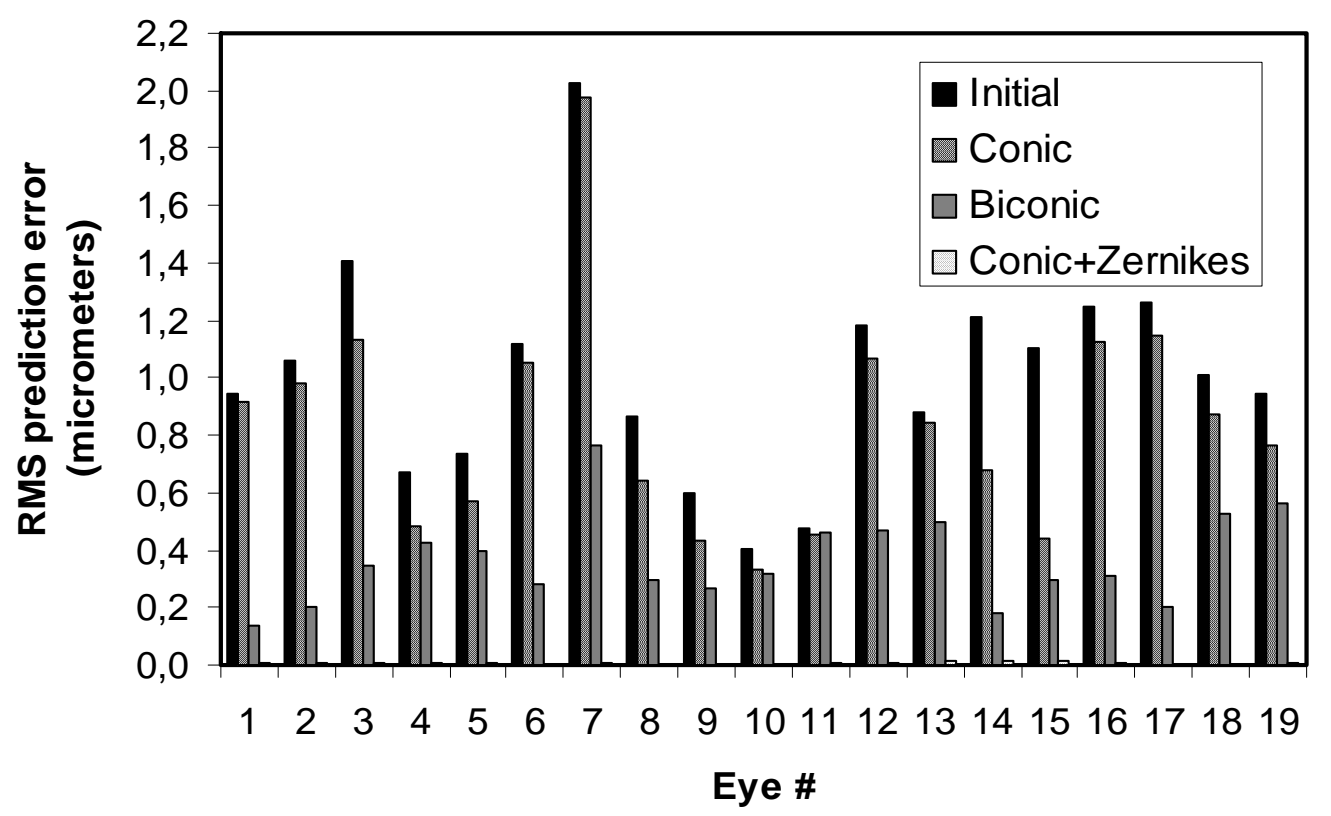

Model \#2

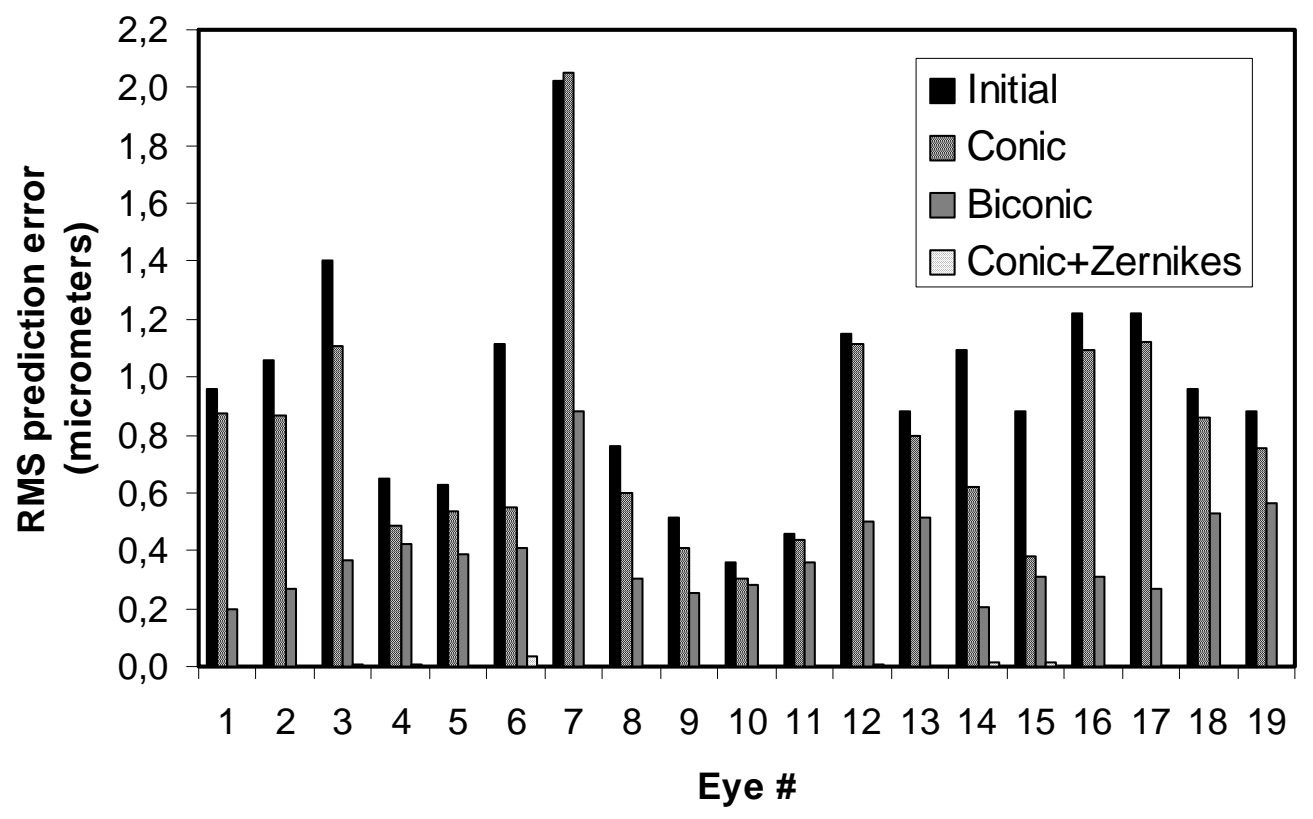

FIG. 2 


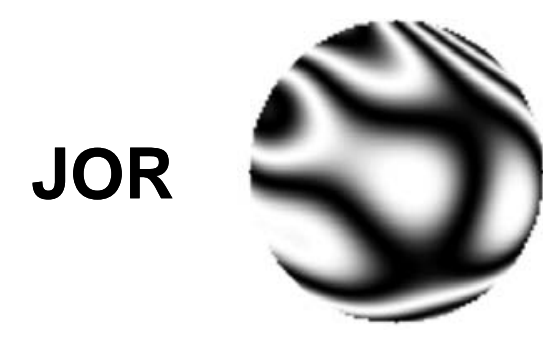

Predictions Model \#1
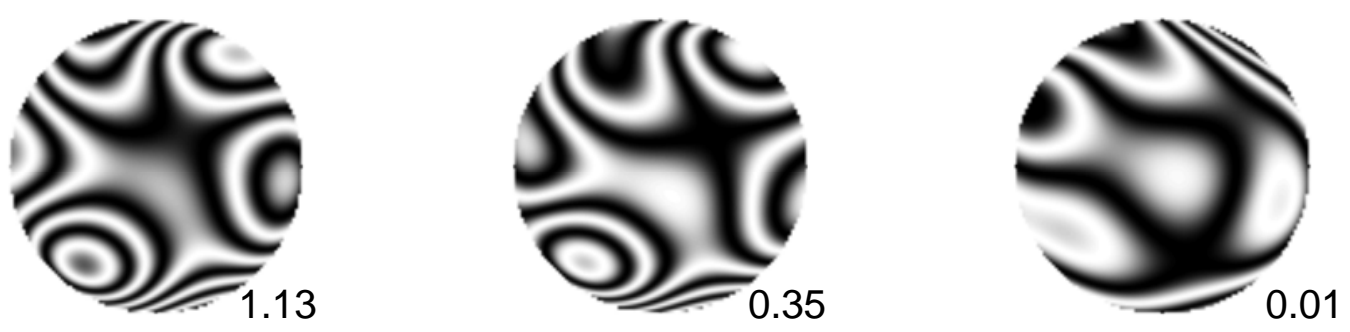

Predictions Model \#2

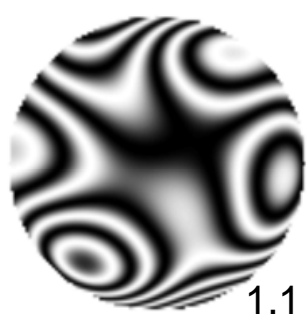

Conic

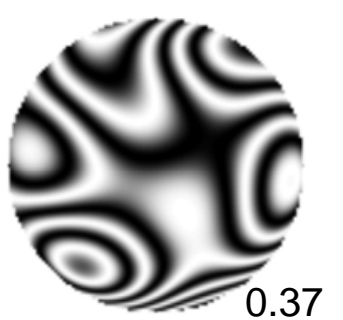

Biconic

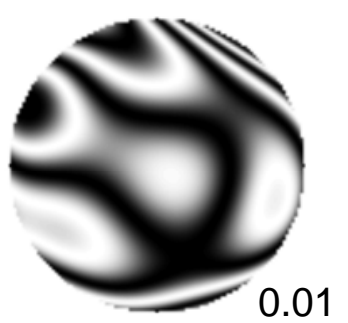

Zernikes

FIG. 3 


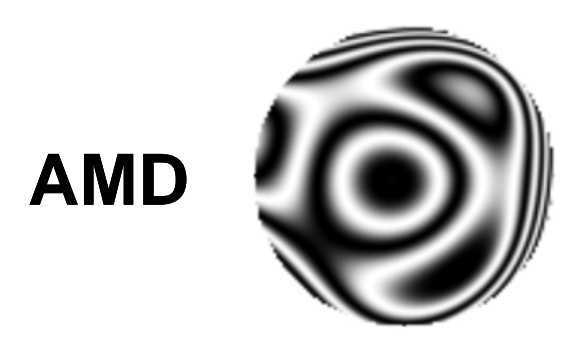

Predictions Model \#1
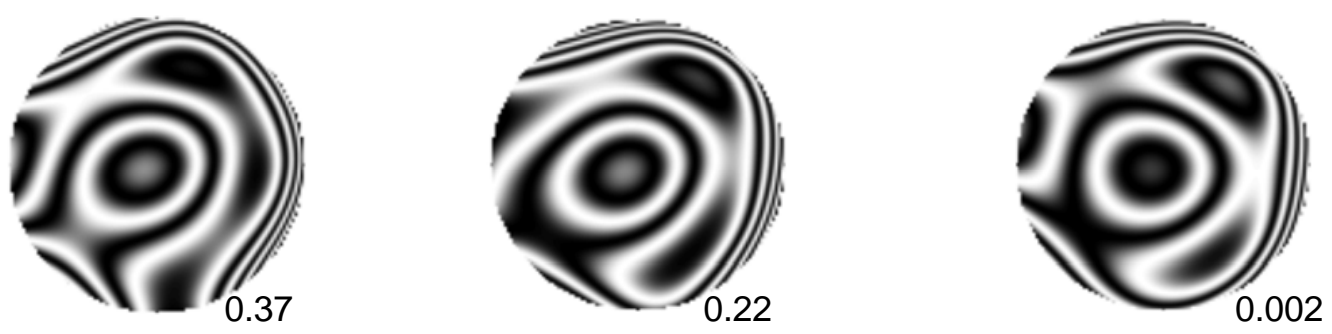

Predictions Model \#2

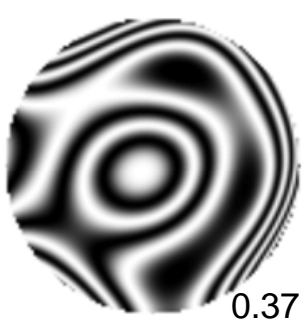

Conic

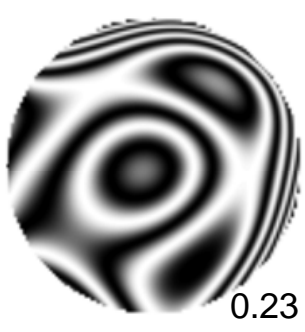

Biconic

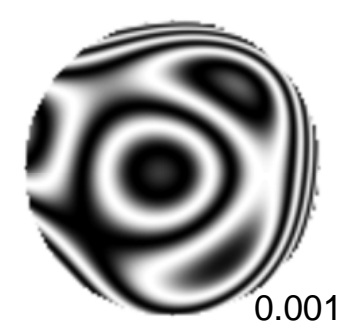

Zernikes

FIG. 4 


\section{Anterior lens radius}

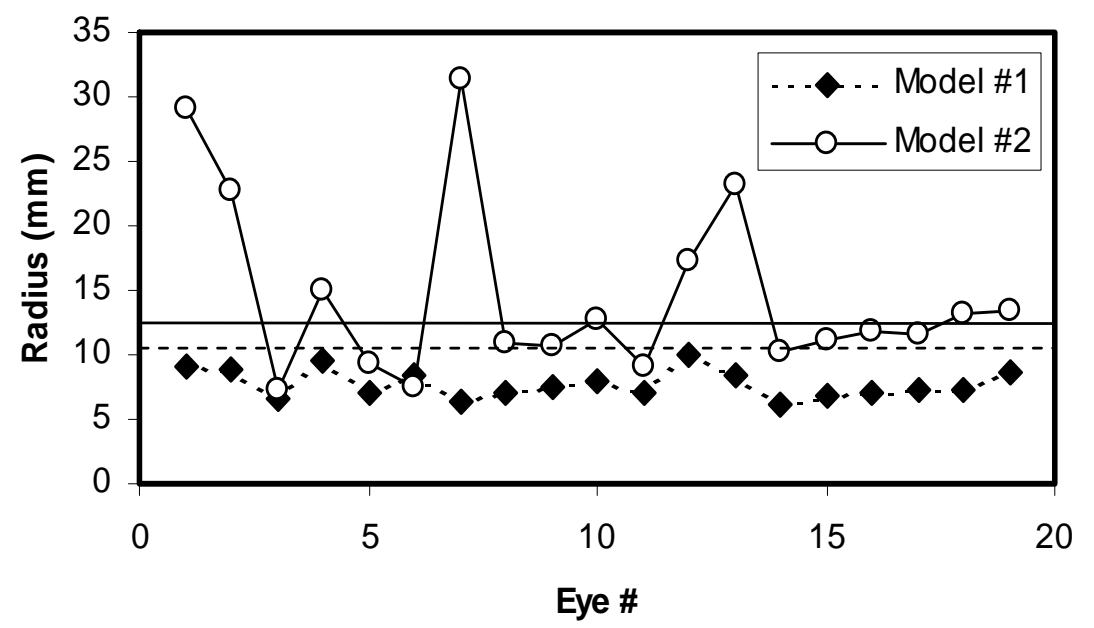

Posterior lens radius

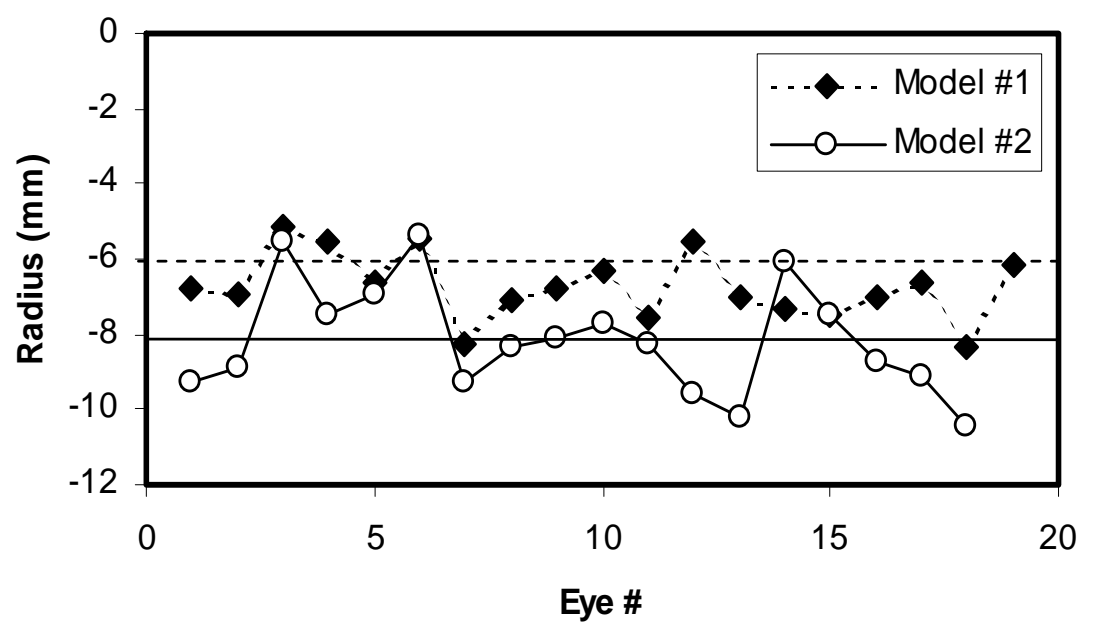

FIG. 5 


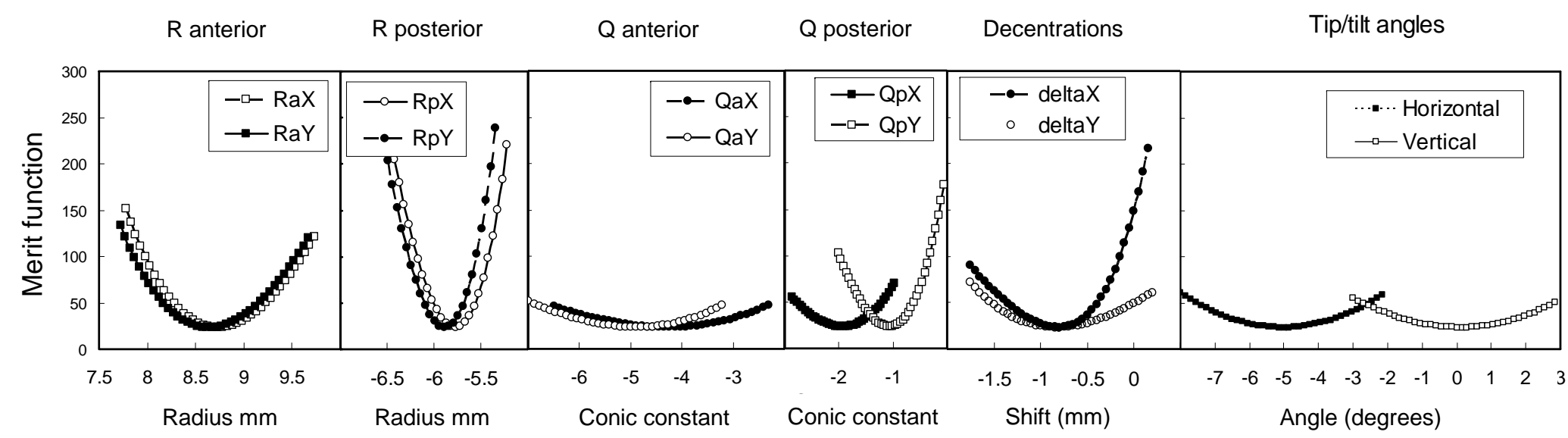

FIG. 6 